\title{
Douleur abdominale chronique : ne pas méconnaître une origine non viscérale
}

\author{
Chronic abdominal pain: consider a non-visceral cause
}

\author{
P. Ducrotté \\ C) Springer-Verlag France 2010
}

Une douleur chronique de topographie abdominale n'est pas obligatoirement synonyme d'une pathologie digestive. Elle peut être la traduction d'une pathologie musculaire abdominale, d'une atteinte des nerfs de la paroi abdominale ou la projection d'une douleur d'origine rachidienne ou costale. Identifier l'origine non viscérale d'une douleur évite la réalisation d'examens complémentaires, en particulier endoscopiques, inutiles et permet de proposer au malade un traitement adapté. L'interrogatoire et l'examen clinique sont essentiels pour orienter le diagnostic. Une composante pariétale pourrait exister dans 10 à $15 \%$ des douleurs abdominales.

Les différents muscles de la paroi abdominale peuvent être douloureux. L'examen recherche un tonus musculaire anormal et l'existence de points « gâchettes douloureux ». La douleur due au muscle psoas est l'une des plus fréquentes et des plus trompeuses. Elle est à l'origine d'une douleur plutôt iliaque, uni- ou bilatérale, parfois accentuée lors de l'effort de poussée exonératrice. Point diagnostique important : quand le psoas est atteint, son raccourcissement lors de l'extension passive de hanche est douloureux. Les points douloureux « gâchettes » pour les muscles droits de l'abdomen peuvent se situer sur tout le trajet du muscle, depuis le rebord costal jusqu'au pubis. Les points douloureux des obliques se détectent au-dessus de la crête iliaque. Les points douloureux diaphragmatiques peuvent être décelés sous l'auvent costal, notamment lors de l'inspiration profonde.

Une douleur pariétale peut être due à un conflit concernant la branche antérieure de l'un des nerfs cutanés abdominaux, au niveau des zones aponévrotiques, le bord externe des muscles droits de l'abdomen (90\% des cas) ou la ligne blanche

\footnotetext{
P. Ducrotté $(\square)$

Département d'hépatogastroentérologie et de nutrition

Hôpital Charles-Nicolle, 1, rue Germont,

F-76031 Rouen cedex, France

e-mail : philippe.ducrotte@chu-rouen.fr
}

entre les droits $(10 \%)$. L'existence d'un signe de Carnett est un élément clé pour le diagnostic de cette douleur toujours très localisée : la contraction des muscles abdominaux induite par la flexion de la tête sur le tronc ou l'élévation des jambes majore la douleur induite par la pression digitale de la zone douloureuse. L'effet antalgique d'une injection anesthésique locale est un second élément en faveur du diagnostic de douleur pariétale.

Une douleur à projection abdominale peut enfin être d'origine osseuse, notamment vertébrale. Elle s'accompagne alors d'une cellulalgie en bande. Schématiquement, une douleur épigastrique doit faire rechercher une atteinte au niveau de T6, une douleur périombilicale une atteinte au niveau T10 et une douleur des fosses iliaques ou sus-pubienne une atteinte de niveau T12-L1. Le syndrome du rebord costal douloureux (syndrome de Cyriax) est dû à une subluxation de l'extrémité antérieure d'une des huitième, neuvième ou dixième côtes ( « côtes flottantes ») qui aboutit à un traumatisme de l'articulation chondrochondrale et à une compression du nerf intercostal correspondant. En cas de retard au diagnostic, la douleur peut devenir chronique et devenir une sensation de brûlure ou de gonflement localisée dans l'épigastre ou l'hypochondre. Le diagnostic du syndrome de Cyriax est clinique : le crochetage de l'auvent costal vers le haut déclenche une douleur vive et élective qui reproduit la douleur spontanée. Le réveil de la douleur est lié au pincement du nerf intercostal par la manœuvre. Un claquement perceptible est parfois le témoin du retour en place du cartilage luxé.

Une origine non viscérale doit être discutée devant une douleur chronique de topographie abdominale. Le temps clinique, interrogatoire et examen clinique, est l'étape clé lors de la première consultation pour orienter vers un tel diagnostic. L'enjeu est d'éviter au malade des examens endoscopiques et/ou radiologiques inutiles à une époque où rationaliser le recours aux examens complémentaires est un souci permanent.

Conflit d'intérêt : aucun. 